\title{
Pneumothorax in Non-Ventilated COVID-19 Patients: A Case Series
}

\author{
Komal Gharsangi ${ }^{1}$, Rajesh Bhawani ${ }^{2}$, Jitender Sandhu ${ }^{3}$ \\ ${ }^{1}$ Assistant Professor, ${ }^{2}$ Prof and Head, ${ }^{3}$ Senior Resident, \\ Department of General Medicine, Shri Lal Bahadur Shastri Govt Medical College, Mandi, \\ Himachal Pradesh-175008 \\ Corresponding Author: Rajesh Bhawani
}

\begin{abstract}
Coronavirus disease 19 (COVID-19) caused by SARS-CoV-2 has spread worldwide resulting in ongoing pandemic. Lung is the most common organ involved in COVID-19 with common radiological finding being ground glass opacities in peripheral distribution and lower lobes. ${ }^{1}$ Pneumothorax is uncommon feature to observe with COVID-19. The exact incidence of this complication is still not known. In a report by Chen et al. $1 \%$ (one patient) had a pneumothorax among other radiographic features. ${ }^{2}$ In a study published by Yang and colleagues in 92 deceased COVID-19 patients, one $(1.1 \%)$ had a pneumothorax. ${ }^{3}$ The mechanism of spontaneous pneumothorax in patients with COVID19 disease is proposed to be related to the structural changes in the lung parenchyma which include cystic and fibrotic changes leading to alveolar tears. In addition to the increase in intrathoracic pressure resulting from prolonged coughing and/or mechanical ventilation. ${ }^{4,5}$ Pneumothorax further complicates the case of COVID-19. We present two cases of pneumothorax in young males, average height, non-smokers without any pre-existing lung pathology.
\end{abstract}

Key words: COVID-19, pneumothorax

\section{CASE PRESENTATIONS}

CASE 1

A 34 year old male, non smoker without any significant medical history was admitted at dedicated Covid health centre for complaints of fever, cough and shortness of breath. Patient was being managed as moderate Covid disease with HRCT chest severity score of 13/25(fig 1). He was in his $7^{\text {th }}$ day of illness when the patient developed increased breathlessness and referred to us which is a Dedicated Covid hospital. On examination pulse rate was $110 / \mathrm{min}$, RR24/min, BP-124/78 $\mathrm{mmHg}$ and $\mathrm{Spo} 2$ of 78 $\%$ on non rebreathing bag mask (NRBM). Chest X-ray of the patient showed left side pneumothorax (fig 2) for which intercostal drainage (ICD) was placed in emergency after which the patient showed symptomatic relief and Spo 2 picking to $92 \%$ on NRBM.
His lab investigations included TLC11700/cumm, D-dimer of $0.3 \mathrm{ug} / \mathrm{ml}$, $\mathrm{CRP}=32 \mathrm{mg} / \mathrm{L}, \mathrm{LDH}=807 \mathrm{U} / \mathrm{L}$. He was managed as per the local guidelines for treatment of COVID-19. The lung gradually expanded but due to persistent air leak had to be kept in situ waiting for surgical intervention.

\section{CASE 2}

A 32 year male patient non-smoker without any co-morbidity was admitted with complaints of cough and shortness of breath since past 5 days. He was tested COVID positive one day back by RT-PCR. On examination his pulse rate was 109/min, $\mathrm{BP}=80 / 50 \mathrm{mmHg}, \mathrm{RR}=40 / \mathrm{min}, \mathrm{Spo} 2=70 \%$ on room air. There was swelling in the left side of the chest and neck region suggestive of subcutaneous emphysema. Clinical 
judgement of left tension pneumothorax was made and in view of urgency ICD was inserted which revealed gush of air and symptomatic relief in the patient. Post ICD insertion x-ray showed expansion of left lung and bilateral opacities (fig 2). He was started on treatment as per local COVID guidelines and high dose steroids. Patient maintained spo 2 of $86-87 \%$ on NRBM for 2 days after which he developed restlessness and respiratory distress. His blood investigations showed TLC of 9400/cumm, $\mathrm{CRP}=35 \mathrm{mg} / \mathrm{L}, \mathrm{D}$ dimer $=0.45 \mathrm{ug} / \mathrm{ml}$. Due to the clinical deterioration of the patient he was put on high flow nasal cannula (HFNC) for respiratory support. As patient was not clinically stable for shifting to CT room, reoeat xray chest was sought which showed increased infiltrates in bilateral lungs (fig 4). The clinical condition did not improve even on HFNC and had to be put on mechanical ventilation next day. Patient had to be given FIO2 of 100 to maintain spo2 of $87-88 \%$. Next day patient had sudden episode of ventricular arrhythmias and went into asystole. Resuscitation was done but could not be revived.

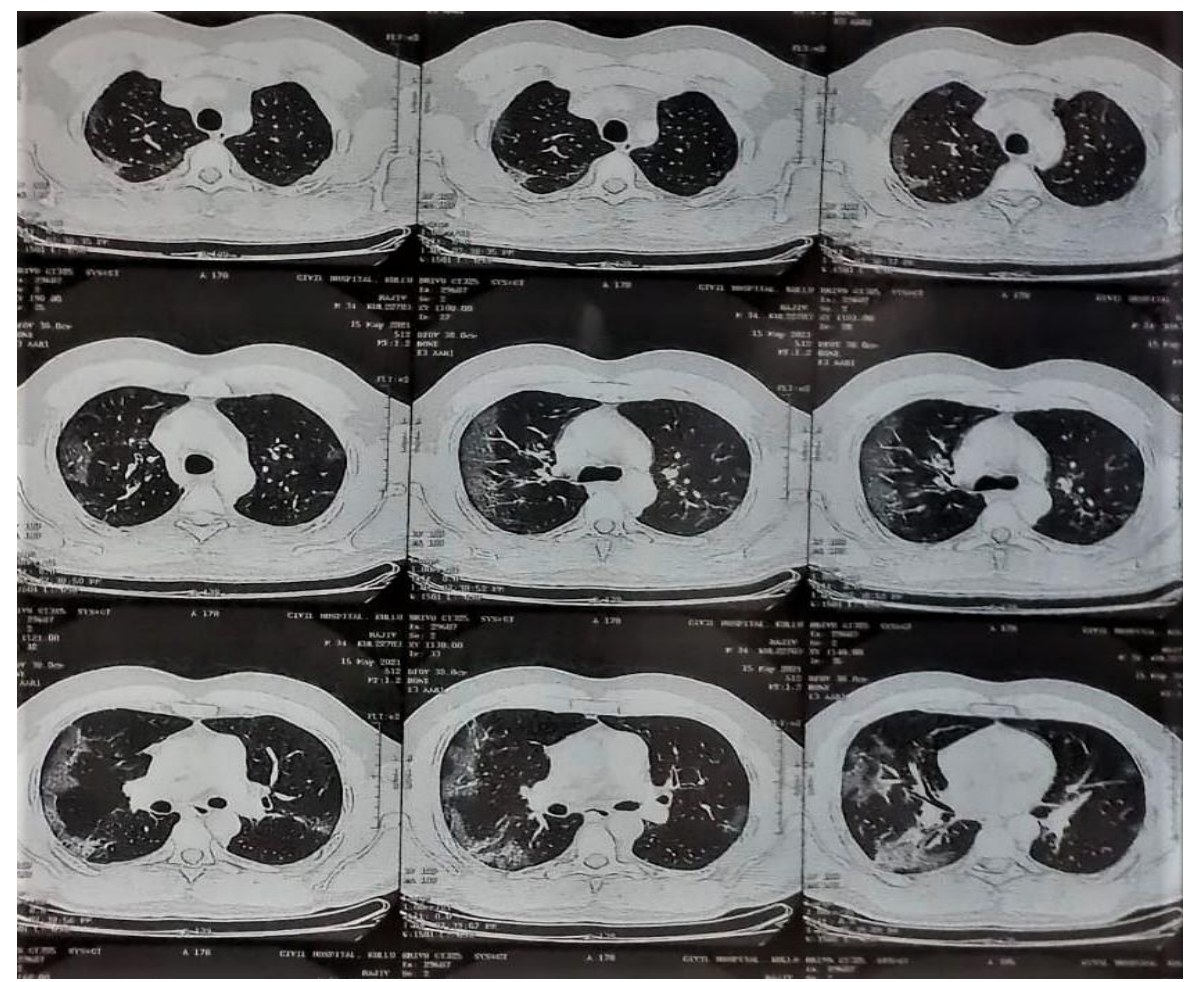

Fig 1 HRCT chest shows ground glass opacities bilaterally on peripheral location

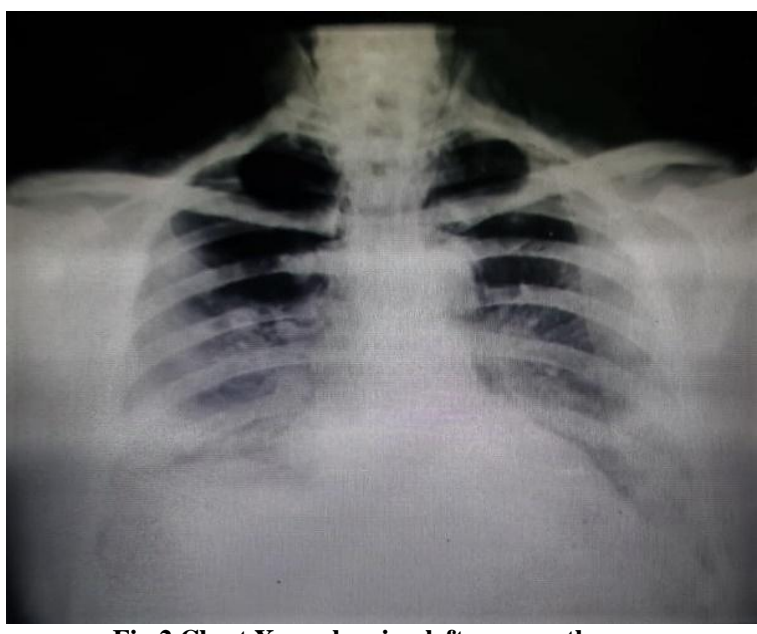

Fig 2 Chest Xray showing left pneumothorax

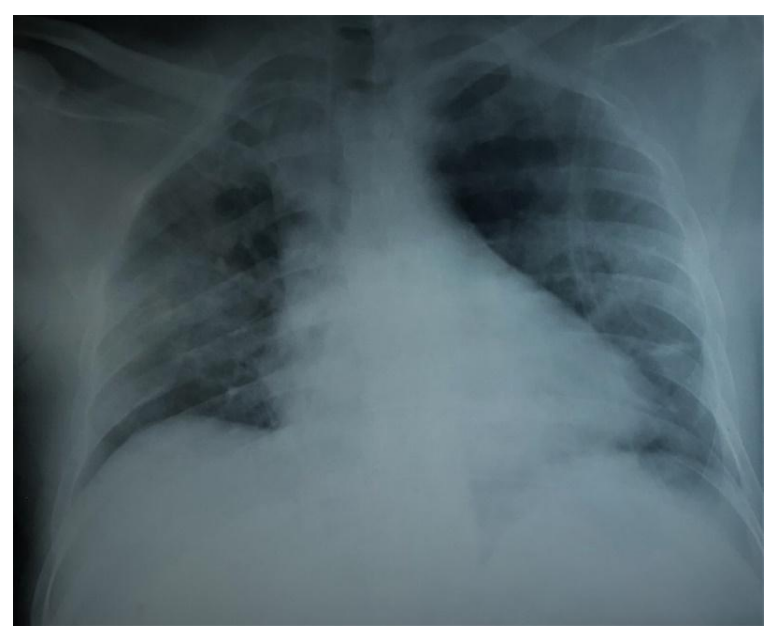

Fig 3 Chest Xray with opacities bilaterally and ICD insitu 


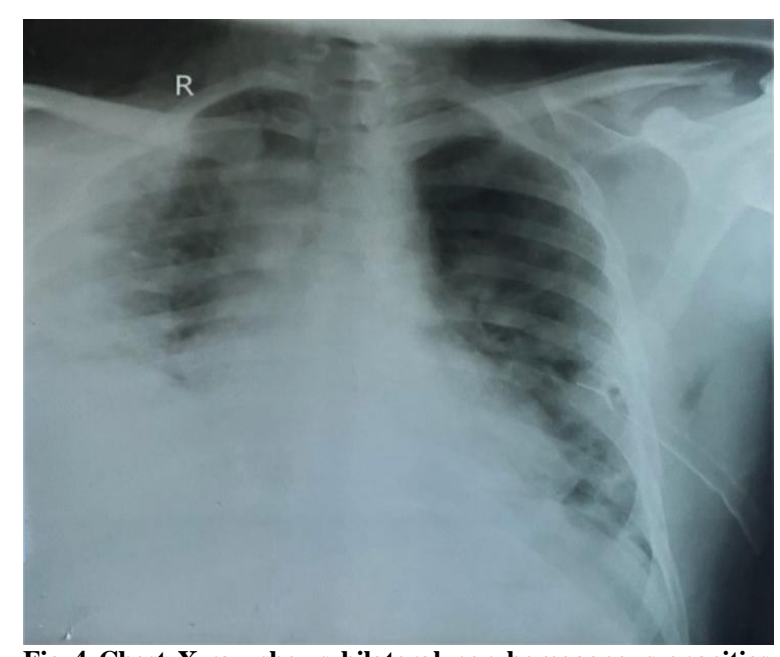

Fig 4 Chest X-ray shows bilateral non-homogenous opacities which has increased on the right lung and ICD in situ on left side

\section{DISCUSSION}

Patients with COVID-19 infection develop severe pneumonia leading to acute respiratory distress syndrome (ARDS) which is characterized radiographically by ground glass opacities, consolidation and fibrotic changes in late stages of the disease. In severe Covid-19 pneumonia, prolonged cough attacks can cause widespread alveolar damage and a sudden increase in alveolar pressure may cause pneumothorax. On a cellular level, there are also immunological changes which may play a role in potentiation of pneumothorax. Studies have suggested that bulla formation in patients with primary spontaneous pneumothorax may be propagated by inflammatory breakdown of elastic fibers. An immune response, mediated by respiratory epithelium, eosinophils, and innate lymphoid cells (ILCs), has been proposed as an aspect of pathophysiology in primary spontaneous pneumothorax. ${ }^{6}$ Mechanical ventilation appears to be a predominant risk factor for development of pneumothorax with COVID-19 pneumonia. Overdistention of the alveoli by mechanical ventilation raises the risk for developing pneumothorax. Wang et al. reported a case of a patient who developed spontaneous pneumothorax, pneumomediastinum and subcutaneous emphysema in a patient who was not on any mechanical ventilation. ${ }^{7}$ Retrospective studies of patients with
COVID-19 suggested that pneumothorax might occur in $1 \%$ of those requiring hospital admission, $2 \%$ in patients requiring Intensive Care Unit (ICU) admission and $1 \%$ of patients dying from the infection. ${ }^{8}$ It has been suggested that the development of pneumothorax during coronavirus infection is a grave prognostic marker. ${ }^{9}$ As in our second case patient was a severe case and with pneumothorax added to the cause of his mortality. A sudden deterioration with rapid oxygen desaturation in a Covid-19 patient could indicate a pneumothorax. Clinicians should be vigilant about the diagnosis and treatment of this complication as it can cause increased mortality and morbidity in COVID patients.

\section{Acknowledgement: None}

\section{Conflict of Interest: None}

\section{Source of Funding: None}

\section{Declaration of Patient Consent}

Appropriate patient consent was taken prior to publication in the journal.

\section{REFERENCES}

1. Li X., Zeng W., Li X. CT imaging changes of corona virus disease 2019(COVID-19): a multi-center study in Southwest China. J. Transl. Med. 2020;18(1):154.

2. Chen N, et al. Epidemiological and clinical characteristics of 99 cases of 2019 novel coronavirus pneumonia in Wuhan, China: a descriptive study. Lancet. 2020;395:507-13

3. Yang F, et al. Analysis of 92 deceased patients with COVID-19. J Med Virol. 2020.

4. Zhou C, Gao C, Xie Y, Xu M. COVID-19 with spontaneous pneumomediastinum. Lancet Infect Dis. 2020;20:510.

5. Liu K, et al. COVID-19 with cystic features on computed tomography: a case report. Medicine. 2020;99:e20175.

6. Kwon BI, Hong S, Shin K, et al. Innate type 2 immunity is associated with eosinophilic pleural effusion in primary spontaneous pneumothorax. Am J Respir Crit Care Med 2013;188:577-85 
7. Wang $\mathrm{W}$, Gao $\mathrm{R}$, Zheng $\mathrm{Y}$, Jiang $\mathrm{L}$. COVID-19 with spontaneous pneumothorax, pneumomediastinum and subcutaneous emphysema. J Travel Med. 2020.

8. Chen N, Zhou M, Dong $X$, et al. Epidemiological and clinical characteristics of 99 cases of 2019 novel coronavirus pneumonia in Wuhan, China: a descriptive study. Lancet 2020; 395: 507-513.
9. López Vega JM, Parra Gordo ML, Diez Tascón A, et al. Pneumomediastinum and spontaneous pneumothorax as an extrapulmonary complication of COVID-19 disease. Emerg Radiol 2020: 1-4.

How to cite this article: Gharsangi K, Bhawani R, Sandhu J. Pneumothorax in non-ventilated COVID-19 patients: a case series. Int $J$ Health Sci Res. 2021; 11(6): 178-181. DOI: https://doi. org/10.52403/ijhsr.20210628 